\title{
THE BLOOD SUPPLY OF THE FLEXOR AND EXTENSOR TENDONS OF THE FINGERS IN MAN
}

\author{
J. G. Brockis, Newcastle-on-Tyne, England \\ From the Department of Surgery, University of Durham
}

The present investigation was inspired by a desire to know something about the factors that produce stiffness of the fingers after injury or inflammation. It seemed logical to inquire whether there were any vascular changes in the fingers in these circumstances, first because in any reaction of a tissue to injury the blood supply is affected, and secondly because fingers that are stiff often undergo so-called trophic changes which may well be related to impairment of blood supply.

Before a study of disease processes could be carried out it was necessary to determine the normal vascular patterns of the tendons and joints of the fingers, more especially as the accounts in the literature are incomplete and sometimes contradictory. This paper deals with the normal blood supply of the tendons and tendon sheaths in man. It is intended in a second paper to describe the changes found in disease and after injury.

Historical note-It was long considered that tendons were avascular structures, and to-day the opinion of Kolliker (1850) is still repeated-that tendons have no blood supply. Ludwig and Schweigger-Seidel (1872) showed that intratendinous vessels existed, as did Mayer (1916). Mayer was chiefly interested in the operative surgery of tendons and gave only limited information about the blood vessels. But he did point out that tendons may receive blood vessels in four regions: 1) at the musculo-tendinous junction; 2) at the osteo-tendinous attachment; 3) in extra-synovial regions through the loose tissue surrounding the tendon (paratenon); and 4) in synovial regions through mesenteric attachments or synovial bands (vincula).

Edwards (1946) has added further to the knowledge of the internal arrangements of the tendon vessels. He showed that the vessels of a tendon are arranged around the collagen bundles of which tendon is composed, and that there is a simple blood-vascular system consisting of a series of longitudinal channels parallel to the collagen bundles, with frequent cross-anastomoses. Each intratendinous longitudinal channel is composed of a small arterial vessel accompanied by two small veins.

\section{MATERIAL AND METHODS}

The material studied has been entirely human; it has included fifty fingers, and several whole hands. Colloidal silver iodide was injected under controlled pressure into the digital arteries. The injection was continued until the silver iodide flowed freely from the veins. At this stage the veins were obstructed by a rubber band and the injection continued for three to four hours to obtain maximal filling of the vessels. Arteries, capillaries and veins were all filled by this technique. The material was dissected, fixed in 10 per cent formalin, developed photographically and then cleared in oil of wintergreen. An alternative method was the injection of Indian ink in plasma. Retrograde venous injection was found to be impracticable because of the presence of valves in the venous system.

\section{OBSERVATIONS}

The feeding vessels of the tendons. Non-synovial regions-Blood vessels reach the tendons in different ways in different parts of the hands and fingers. The simplest arrangement is 
found in the distal part of the palm and in the forearm, where the flexor tendons have no tendon sheaths but lie in loose connective tissue (paratenon). Here vessels pass through the loose tissue at frequent intervals to supply the tendon (Fig. 1). They are arranged in curves

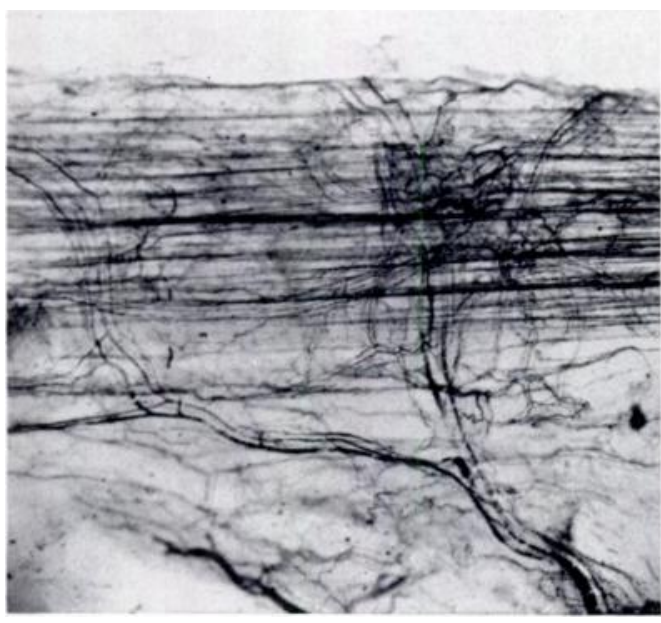

FIG. 1

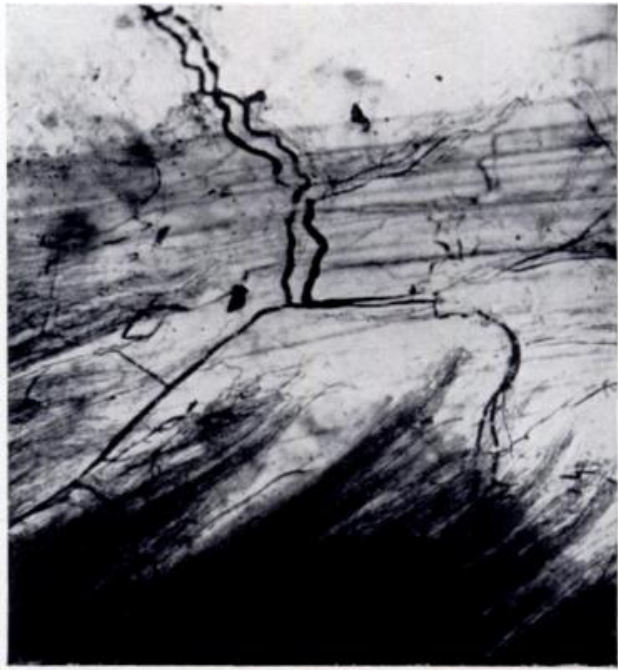

Fig. 2

Figure 1-Flexor tendon in an extra-synovial region showing blood vessels passing to it through the loose connective tissue (paratenon) surrounding it $(\times 7)$. Figure 2-. Iusculo-tendinous junction of flexor digitorum sublimis showing vessels passing to the muscular and tendinous parts $(\times 9)$.

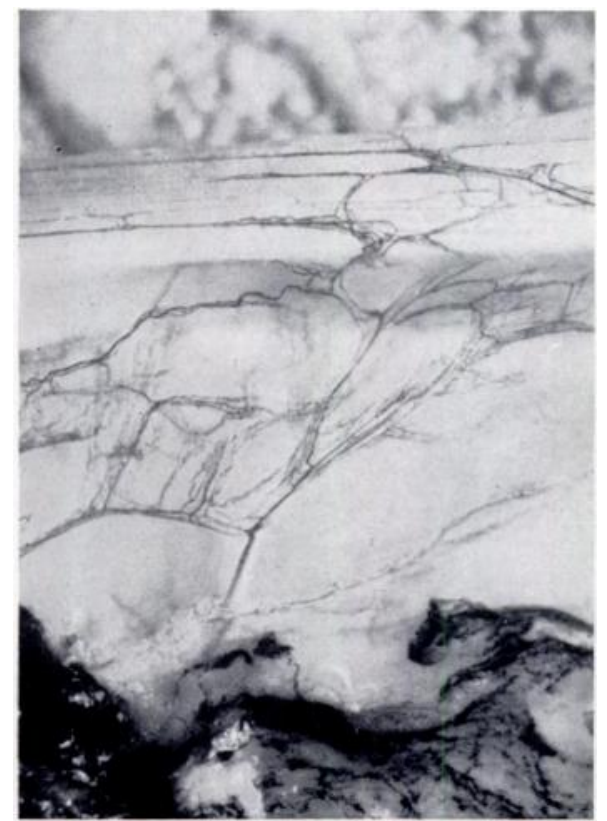

Fig. 3

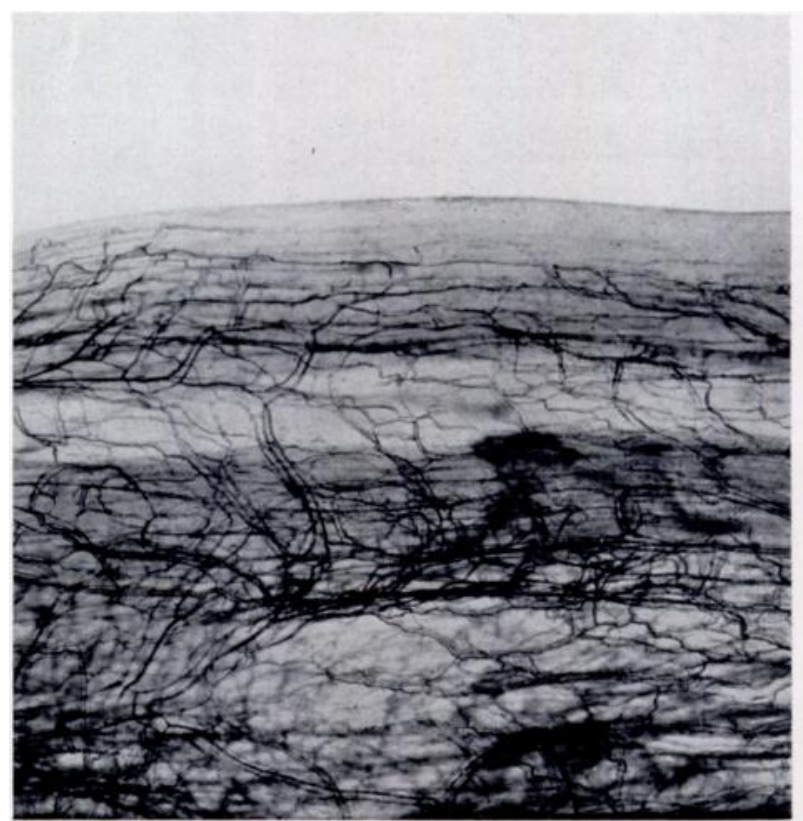

FIG. 4

Figure 3-Flexor digitorum sublimis tendon of the little finger showing the vascular arcades within the palmar bursa $(\times 5)$. Figure 4-Two flexor digitorum profundus tendons lying within a reflection of the palmar bursa. Vessels entering from the proximal and distal ends of the bursa $(\times 6 \cdot 5)$.

so that when the tendon moves the curves are straightened out. The connecting vessels consist of an artery and two small accompanying veins, and there are frequent cross-connections between the veins. Usually one is considerably larger than its fellow and the diameter of 
the veins is slightly greater than that of the artery. The extensor tendons are also largely without a synovial sheath, and are covered with paratenon, through which many ressels pass at frequent intervals to the tendon. The arrangement is similar to that found in other areas where there is no synovial covering.

In the region of the musculo-tendinous junction there are numerous feeding vessels which in some instances divide into two-one branch passing into the muscle and the other into the tendon (Fig. 2). Other vessels pass directly from the muscle belly into the tendon. The arrangement is in all respects similar to that found where the tendon has no synovial sheath.

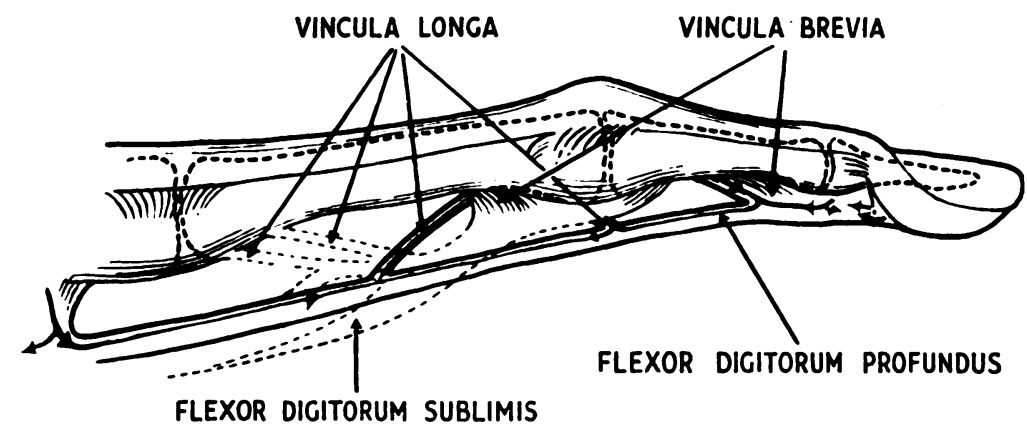

FIG. 5

Diagram of a main vascular channel of the flexor digitorum profundus tendon within the digit.

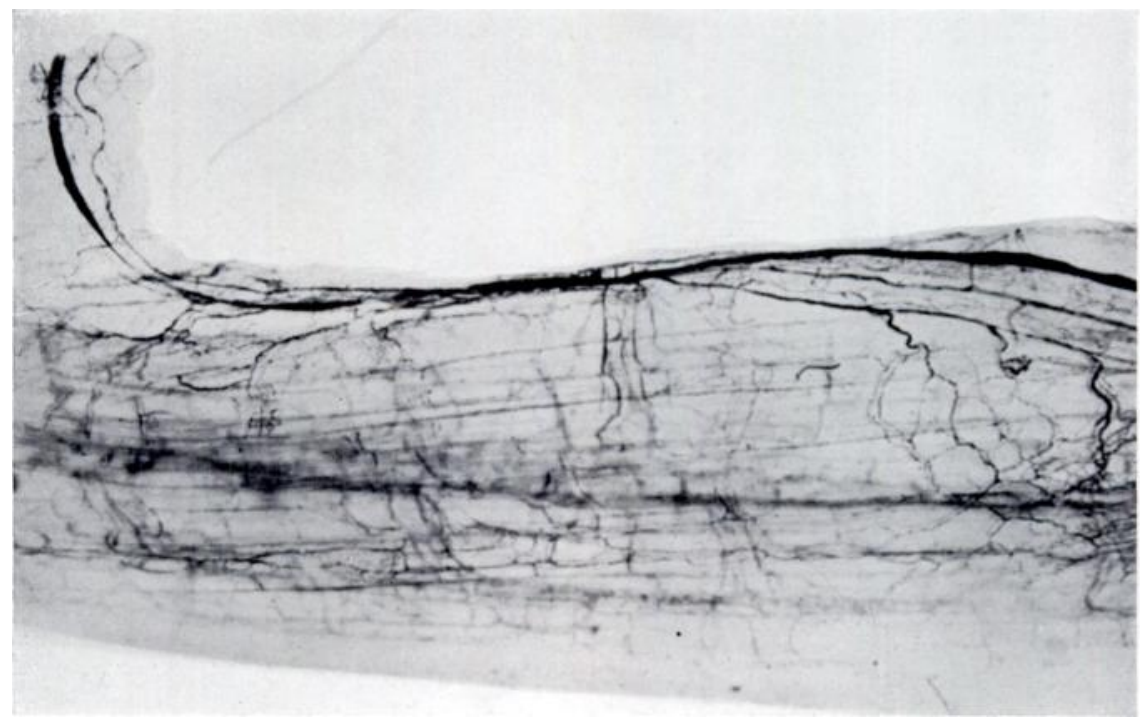

FIG. 6

Main vascular channel of a flexor digitorum profundus tendon within the synovial digital sheath $(\times 10)$.

Synovial regions-Where the tendon lies within a synovial sheath the supplying blood vessels follow definite patterns. In the palm the flexor digitorum sublimis and the flexor digitorum profundus tendons lie within the palmar bursa. The four tendons of the latter lie parallel to one another on the deep aspect of the flexor digitorum sublimis. Although all the long flexors of the fingers lie within reflections of the palmar bursa, each of the tendons of the flexor digitorum sublimis has an incompletely separated mesotendon of its own. Between the visceral layers of the palmar bursa and the tendons there is a certain amount of loose connective tissue which is continuous both above and below the bursa with the paratenon.

VOL. 35 B, NO. 1, FEBRUARY 1953

$$
\mathrm{I}-\mathbf{I}_{(8)}
$$


The ressels that supply the tendon run in this loose connective tissue, and make many connections with those within the tendon substance. Through this loose connective tissue there is a sliding plane between the tendons and the visceral layer of the bursa; the connecting vessels are arranged to allow for this movement.

The flexor digitorum sublimis tendons are supplied by feeding vessels which form arcades between the two visceral layers of the bursa (Fig. 3), similar to those seen in the mesentery of the small intestine. The arcades are usually three in number and from the most distal row connections are made with the intratendinous vessels.

In the palmar bursa, the flexor digitorum profundus tendons lie together in connective tissue, sandwiched between two layers of visceral synovial membrane. The vessels supplying the tendons ramify in this tissue (Fig. 4), entering both at the proximal and distal ends of the bursa.

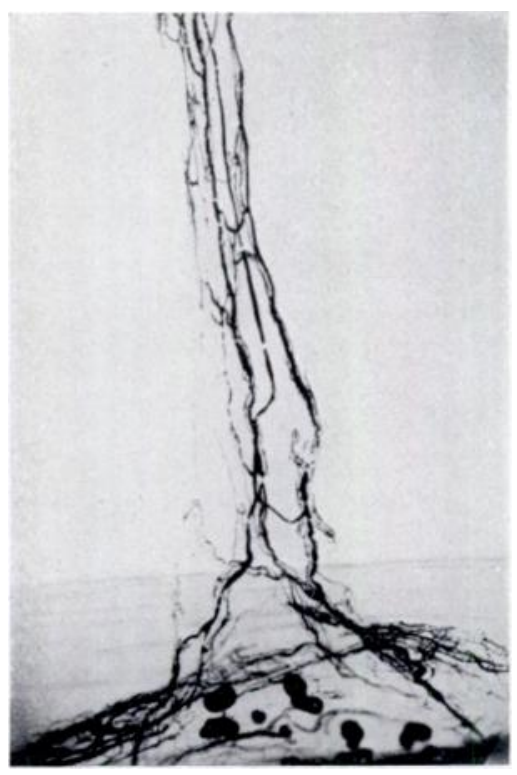

Fig. 7

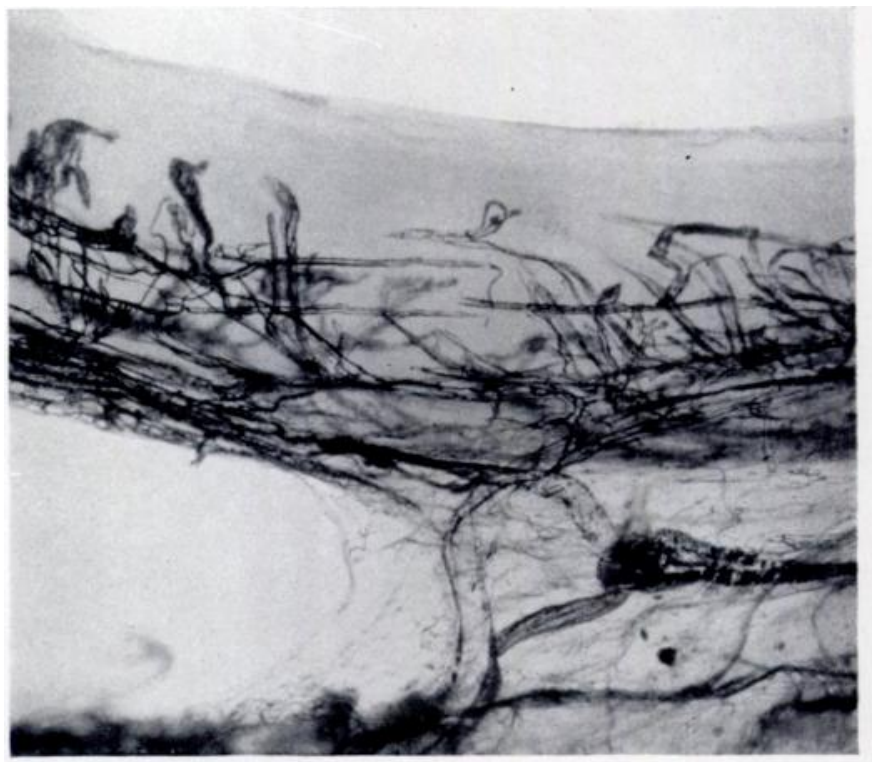

FIG. 8

Figure 7-Blood vessels of the vinculum longum of a flexor digitorum profundus tendon $(\times 15)$. Figure 8-Vinculum brevium containing the main vascular channel close to the insertion of the flexor digitorum profundus tendon.

The blood supply to the tendons within the digital sheath differs from the arrangement in the palm. The visceral layer of the synovial membrane is closely adherent to the tendons without any loose intervening connective tissue, except in sites where friction is minimal. Vessels can only pass to and from the tendons at their insertions, through synovial bands (vincula) or at the points of reflection of the synovial sheath on to the tendons at the proximal and distal ends of the digital sheaths. There is a constant pattern of vessels supplying the tendons within the digital sheath. On the dorsum of the flexor digitorum profundus tendon there is a main longitudinal channel which originates in the palm and joins the tendon on its radial side. This channel rapidly attains a mid-dorsal position in which line the vessels continue as far as the insertion of the tendon (Fig. 5). The vessels comprise a small artery and two veins, surrounded by a little loose connective tissue deep to the synovial membrane (Fig. 6). It is likely that this is a position of minimal friction, and it is only in this dorsal position that the synovial membrane is not firmly bound down to the tendon. Joining this longitudinal channel at intervals are tiny vessels which run in the vincula (Fig. 7). These feeding vessels not only connect with the main channel but also send branches into the tendon 
substance. Near the insertion of the profundus tendon into the distal phalanx the main dorsal channel turns along the synovial reflection (vinculum brevium) to join with transverse vessels which cross the neck of the intermediate phalanx (Fig. 8). From the vessels in this reflection branches pass towards the tendon insertion, which is also supplied by branches

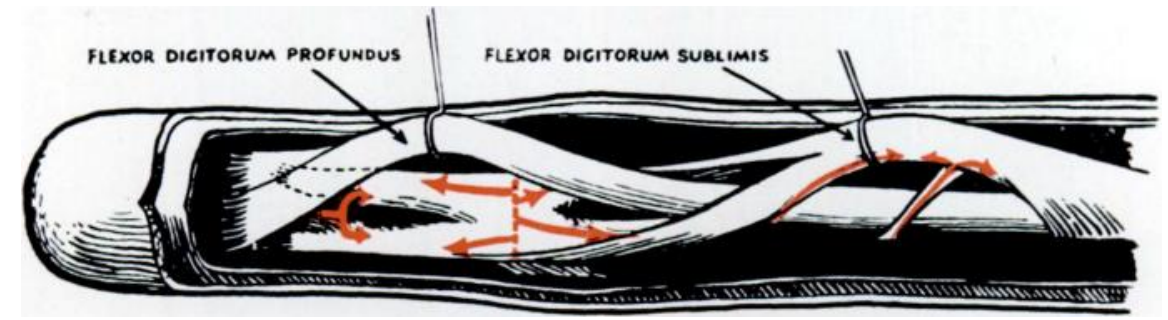

FIG. 9

Diagram of the main vascular channels of the intrathecal part of a flexor digitorum sublimis tendon.

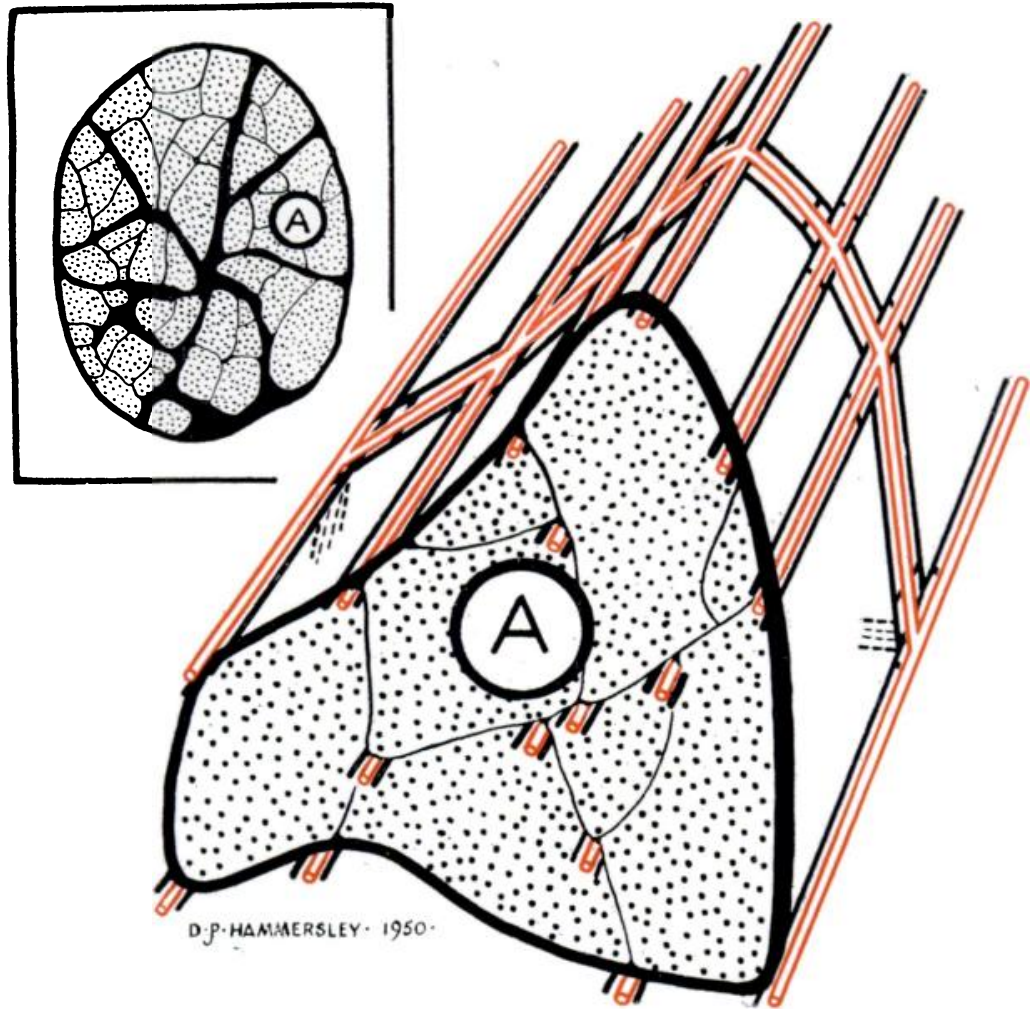

FIG. 10

Cross-sectional diagram of the vascularisation of a trpical tendon showing the position of arteries and veins.

from the capsular vessels at the base of the distal phalan:. Comparatively few periosteal vessels take part in supplying blood to the tendon.

The flexor digitorum sublimis tendons also have main vascular supply channels, each comprising an artery and two veins, but the arrangement is more complicated than in the profundus tendons because of the way in which the flexor digitorum sublimis divides into two slips over the proximal phalanx. These slips pass on either side of the flexor digitorum profundus tendon to reach the anterior surface of the proximal interphalangeal joint, where 
they join again. Here the innermost fibres decussate. Finally two slips separate to be inserted into the sides of the intermediate phalanx. The vascular system of the intrathecal part of the flexor digitorum sublimis centres around the proximal interphalangeal joint. Between

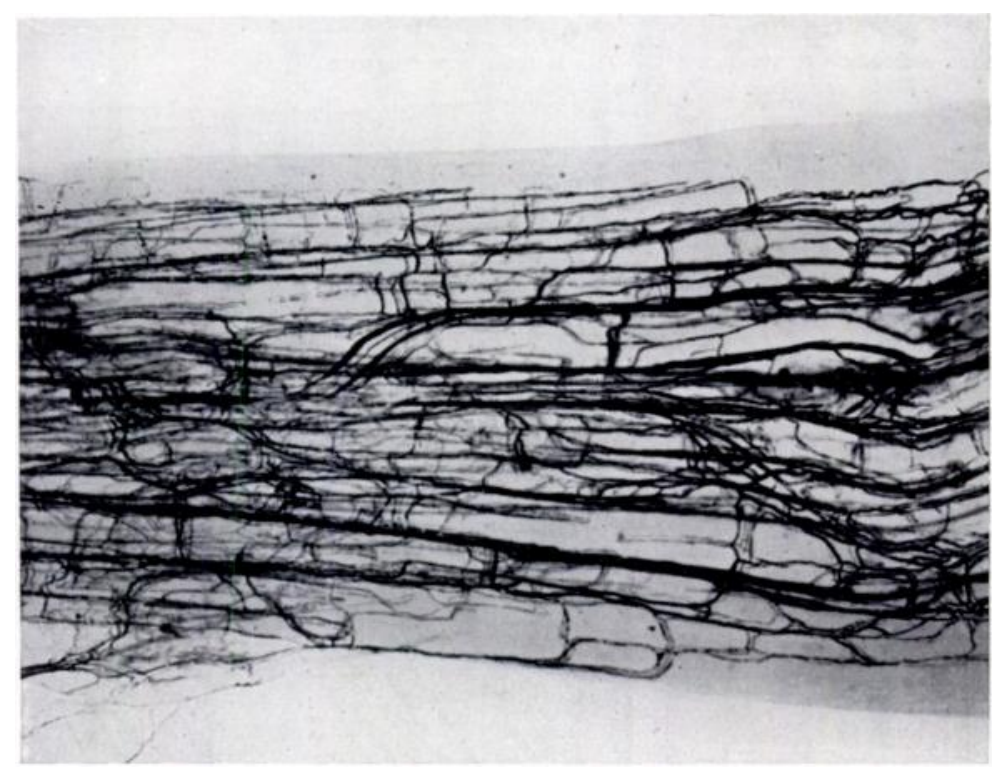

FIG. 11

Low power view of flexor digitorum profundus tendon rendered transparent to show the intratendinous vessels $(\times 9)$.

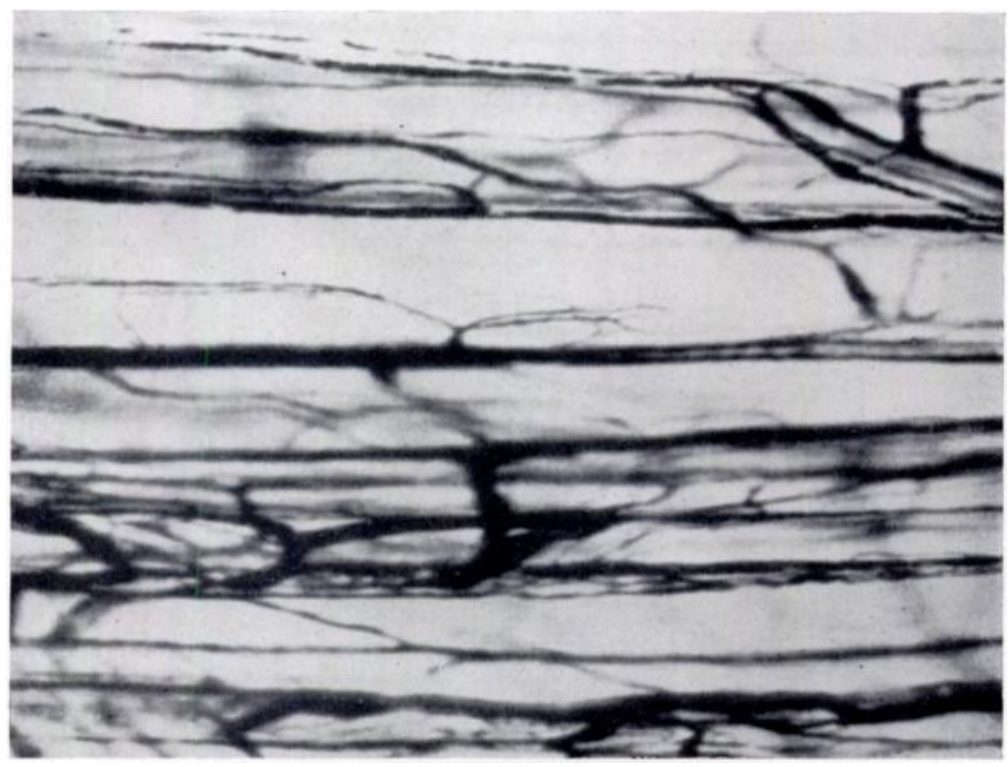

FIG. 12

Cleared specimen of flexor digitorum sublimis tendon to show intratendinous vessels $(\times 38)$.

the two digital arteries at this level there are connecting vessels which also supply the capsule. From them vessels pass distally and proximally. A branch follows each slip of insertion to its termination in a distal direction. The second branch passes proximally around the flexor 
digitorum profundus tendon with the slip of the flexor digitorum sublimis. These vascular channels are arranged in the form of a letter " $\mathrm{H}$ " centred over the proximal interphalangeal joint (Fig. 9). The channel which runs proximally receives communicating vessels from a

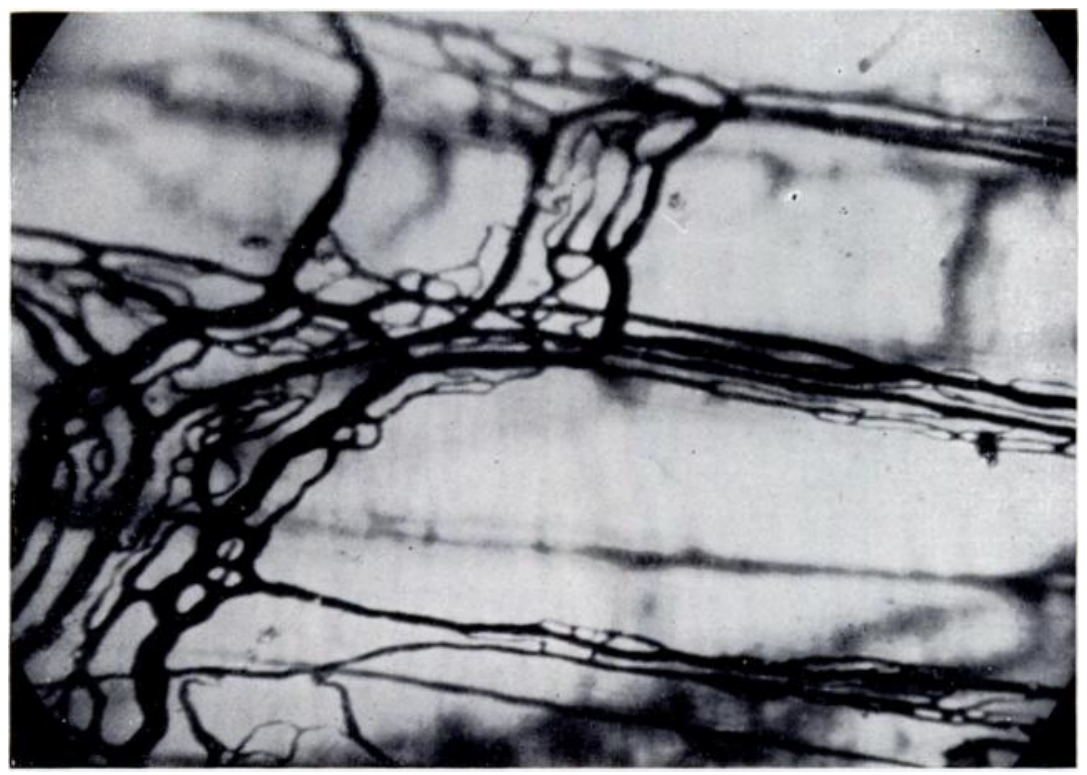

FIG. 13

Higher power view of two arteries, veins and capillaries arranged around two collagen bundles $(\times 95)$.

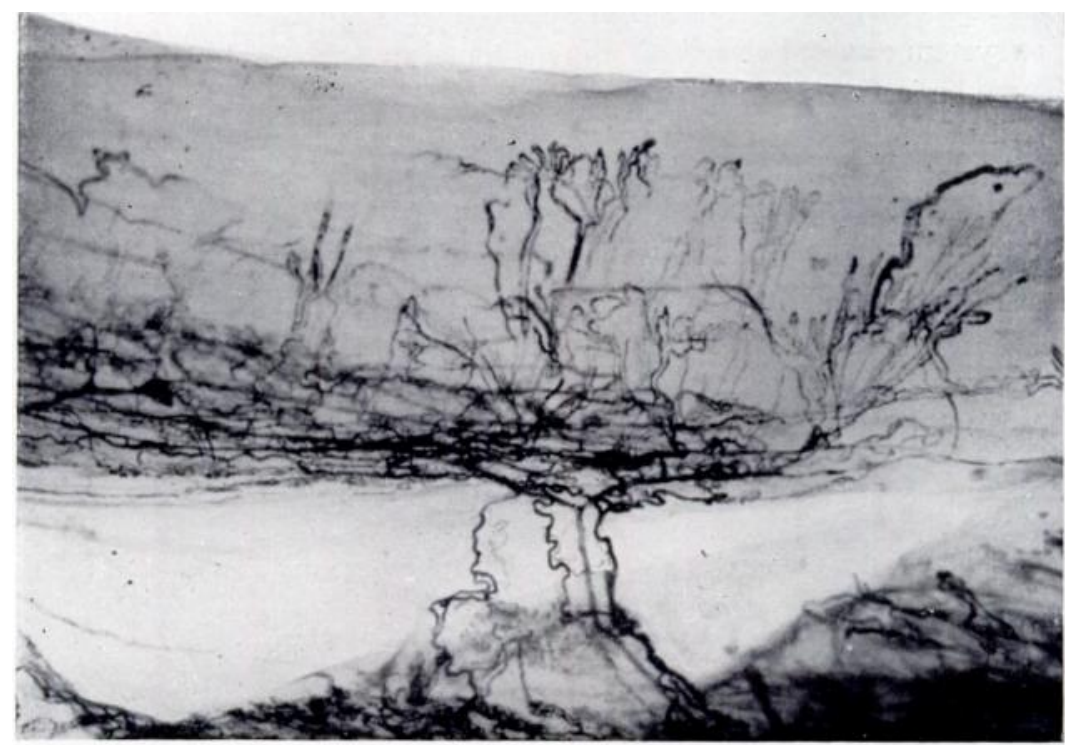

FIG. 14

Lateral view of cleared specimen of the flexor digitorum profundus tendon at the level of the proximal interphalangeal joint $(\times 10)$.

vinculum longum on the lateral edge of the slip. The arrangement is such that these vascular channels lie in positions of minimal friction.

Intratendinous vascular patterns--The vessels within the tendon lie in the interfascicular 
connective tissue, which binds the collagen bundles together. The collagen bundle of fibres appears to be the vascular unit around which the blood vessels are arranged; arteries, veins and capillaries are all to be found in the interfascicular connective tissue but no blood vessels are demonstrable inside the individual collagen bundles (Fig. 10).

The blood-vascular system within the tendons of the hand consists of large numbers of longitudinal vessels lying parallel to the collagen bundles (Figs. 11 to 13). Each arteriole is accompanied by two venules: one venule is usually larger than the other and they communicate at frequent intervals. There are thus many more venous than arterial channels. The longitudinal intratendinous vessels connect with one another through transverse branches, which in turn connect with the main longitudinal vascular channel of the tendon.

This simple blood-vascular pattern is found throughout the flexor and extensor tendons of the hand with two exceptions, both of which occur in the flexor digitorum profundus tendon. In this tendon the longitudinal intratendinous pattern is upset at the level of the proximal and distal interphalangeal joints. Over the proximal interphalangeal joint, vascular loops are to be seen passing vertically through the tendon substance. These loops originate from either the main vascular longitudinal channel on the dorsal surface of the tendon or from the vascular twigs which pass to the tendon in the synovial bands (vincula longa). It may well be that the orientation of the vessels in a vertical direction in this region is related to the fact that the tendon is bent to a greater degree than any other tendon in the body during flexion of the proximal interphalangeal joint: so that if the vessels were not so arranged, the blood flow in the tendon at this point would be obstructed by flexion of the finger (Fig. 14).

Over the distal interphalangeal joint the regular vascular pattern is again disturbed (Fig. 8). At this site vessels reach the tendon along the vinculum brevium, and many branches arise which pass through the tendon to join the fine longitudinal vessels running between the collagen bundles.

\section{SUMMARY}

The blood supply of the flexor and extensor tendons of the fingers is described. The blood-vascular system consists of main feeding channels which supply a longitudinal network of vessels. These lie in the interfascicular connective tissue.

A variation of the typical intratendinous vascular pattern in relation to the proximal interphalangeal joint is described.

I should like to thank Professor F. H. Bentley and Mr F. Braithwaite for their helpful criticism of this work, Mr Wilson for the histological preparations, Messrs Ridley and Leslie for the photography and Mr D. P. Hammersley for the illustrations.

\section{REFERENCES}

Edwards, D. A. W. (1946): The Blood Supply and Lymphatic Drainage of Tendons. Journal of Anatomy, 80, 147.

Kölliker, A. von (1850): Mikroskopische Anatomie oder Gewebelehre des Menschen. Leipzig: W. Engelmann.

Ludwig, C., and Schweigger-Seidel, F. (1872): Die Lymphgefässe der Fascien und Sehnen. Leipzig: Hirzel.

MaYer, L. (1916): The Physiological Method of Tendon Transplantation. Surgery, Gynecology and Obstetrics, 22, 182, 298, 472. 